\title{
Evaluación de conocimientos médicos: diagnóstico de afecciones dermatológicas prevalentes
}

\author{
A. Santos Jr., M.G.G. Andrade, A.M.B. Zeferino, S.M.R.R. Passeri, E.M. Souza, P.E.N.F. Velho
}

Objetivo. La enseñanza de la dermatología es a menudo poco apreciada, a pesar de las frecuentes afecciones dermatológicas en la práctica clínica. El objetivo de este estudio es valorar los conocimientos de médicos no dermatólogos y de estudiantes del curso de medicina de la Facultad de Ciencias Médicas de la Universidad Estatal de Campinas, Brasil, en el tratamiento de pacientes con afecciones dermatológicas. Entre los estudiantes había 24 que integraban un nuevo modelo curricular, después de la reforma educativa, lo que permitió un mayor número de actividades relacionadas con la especialidad. Sujetos y métodos. Este estudio se desarrolló a través de un examen escrito con diez casos clínicos y sus imágenes. El sujeto evaluado debía marcar una de las cinco alternativas para cada pregunta. Este instrumento se administró a 83 médicos y 55 estudiantes de medicina, de forma anónima. Entre el grupo de médicos, ocho eran especialistas en Dermatología y sirvieron como referencia para el estudio. Resultados. Los estudiantes del nuevo plan de estudios obtuvieron un promedio de 6,34 en relación con la puntuación de los dermatólogos, considerada de 10, la más alta posible. La puntuación promedio de los otros estudiantes fue de 3,82, y la de los médicos no especialistas de las unidades básicas, de 3,32. Conclusiones. El estudio permitió demostrar que los cambios en la enseñanza de la Dermatología fueron importantes para la adquisición de conocimientos de la especialidad por los alumnos que siguieron el nuevo currículo de la facultad y para asumir que los médicos no especialistas del Programa de Salud Familiar que prestan servicios en atención primaria de las regiones estudiadas no recibían formación suficiente para el ejercicio de la especialidad en la calidad de médicos con formación general.

Palabras clave. Currículo. Cuidados médicos. Dermatología. Educación. Enseñanza. Valoración.
Evaluation of medical skills:

\section{diagnosis of prevalent dermatological lesions}

Aim. Instruction in dermatology is often under-valued, in spite of the frequency of dermatological complaints in clinical practice. The aim of this study is to evaluate the skill of doctors who are not dermatologists as well as the students of the Medical School at the Campinas State University, Brazil, in the treatment of patients with dermatological lesions. Among the students, there are 24 participating in a new model of curriculum, which came as a result of a revision of teaching methods, which allows a greater number of activities relating to this specialty. Subjects and methods. This study was designed by using a writing test which contains ten clinical cases and the images relating to these cases. The person being evaluated was required to choose one of five alternatives for each question. This instrument was applied anonymously to 83 doctors and 55 medical students. In this group of doctors, eight were specialists in dermatology and served as a point of reference for the study. Results. Students of the new curriculum had an average of 6.34 considering the note of dermatologists as ten, the maximum score possible. The average of the other students was 3.82 and the average of the non-specialist doctors attending in primary care units was 3.32. Conclusions. The research demonstrated that the change in teaching of dermatology was a relevant factor for the acquisition of knowledge in this area in the new curriculum at the university. It also supported what had been previously supposed, that non-specialists in the Family Health Program who attend patients at health primary care in the regions evaluated did not receive adequate training for the performance of this specialty as expected to general practitioners.

Key words. Assessment. Curriculum. Dermatology. Education. Medical care. Teaching.
Facultad de Ciencias Médicas. Universidad Estatal de Campinas (Unicamp). Campinas, SP, Brasil.

\section{Correspondencia} Dr. Paulo Velho. Departamento de Clínica Médica. FCM/Unicamp. Cidade Universitária Zeferino Vaz, s/n. CP 13081 970. Campinas, SP, Brasil.

E-mail:

pvelho@unicamp.br

Financiación: PIBIC / CNPq. 


\section{Introducción}

El número de pacientes que buscan atención médica dermatológica es elevado: las lesiones de la piel afectan entre un tercio y una cuarta parte de la población y el $10-15 \%$ de las consultas de los médicos generales se relacionan con la piel $[1,2]$. En el año 2005, las enfermedades dermatológicas representaron el 8,84\% de las consultas de los adultos y el 9,96\% de las consultas pediátricas en un Servicio de Urgencias chileno [3]. Entre los pacientes ambulatorios pediátricos se estima que el $15-30 \%$ de las consultas se deben a una enfermedad de la piel y el nivel de atención primaria podría resolver el 90\% de esta demanda [4]. En un estudio realizado en Campinas, aproximadamente uno de cada diez usuarios de las unidades básicas de salud (UBS) se quejaba de una afección dermatológica, y uno de cada cuatro usuarios presentaba un signo u síntoma dermatológico que requería atención médica [5].

El número de lesiones de esta rama del conocimiento médico es muy elevado y existen varios diagnósticos diferenciados para una misma lesión. Basándose en la observación detallada, el examen dermatológico permite llevar a cabo el razonamiento diagnóstico. Sin embargo, para alcanzar este objetivo, se necesita tiempo para aprender a identificar y diferenciar las lesiones cutáneas de base, además del conocimiento de los amplios contenidos académicos [6]

Las reformas curriculares en la educación médica, que ocurren en todo el mundo, conllevan la reflexión sobre la suficiencia, o no, del tiempo dedicado a cada área en la formación del profesional con conocimientos generales, y la Dermatología se inscribe en este contexto. En relación con la enseñanza de la Dermatología en cursos de licenciatura en medicina, en los programas de residencia médica y de medicina de familia, el tiempo destinado al aprendizaje es limitado $[7,8]$. Posiblemente, como resultado, algunos estudios indican que los profesionales de la salud de diversos lugares no dominan los contenidos previstos para el correcto ejercicio de la profesión [9-15].

En las escuelas de medicina del Reino Unido, actualmente, el tiempo promedio en el currículo de licenciatura que se dedica a la dermatología es de nueve días ( $54 \mathrm{~h}$ ), con un rango que varía entre 4-15 días. Estos valores son similares a los de los años ochenta en el mismo país (promedio: 53 h; rango: 21-123 h). La Asociación Británica de Profesores Universitarios de Dermatología (BAUTOD) recomienda un mínimo de diez períodos $(30 \mathrm{~h})$, pero preferiblemente más $[2,16]$.

En 2001 comenzó el nuevo plan de estudios en la Facultad de Ciencias Médicas de la Universidad Estatal de Campinas (FCM/Unicamp), que busca formar médicos humanistas, críticos y reflexivos, capaces de ejercer un papel de liderazgo, de mantenerse al día y comprender las cuestiones de gobierno y de gestión, trabajando en equipo.

Los cambios implementados desde el primer año de la escuela de medicina permitieron aumentar el tiempo dedicado a la enseñanza de la Dermatología. Estos cambios tenían por objeto mejorar la formación de médicos en la especialidad. Desde el tercer año del nuevo plan de estudios, los estudiantes tienen contacto con Dermatología Clínica en el módulo de Fisiopatología Integrada. En el cuarto año se desarrollan actividades prácticas de observación en las clínicas de la especialidad, ubicadas en el Hospital de Clínicas, y un contenido teórico. En el quinto año del nuevo plan de estudios, los alumnos tratan a pacientes con dermatosis en UBS de la región.

Con la reforma curricular, la disciplina de Dermatología mantuvo su contenido teórico, pero hubo un aumento en las actividades prácticas desde el cuarto año.

Antes, los estudiantes aprendían un contenido de Dermatología en el tercer año impartido por profesores del Departamento de Anatomía Patológica, un contenido teórico enseñado en el cuarto año, $y$ siete periodos obligatorios de practica observacional, en los dos últimos años del curso. En el nuevo plan de estudios, comienza a enseñarse el contenido de Dermatología Clínica, conjuntamente con los patólogos anatómicos y patólogos clínicos, en el tercer año. En el cuarto año, además de contenidos teóricos, los estudiantes tienen 30 horas de actividades con los pacientes o con imágenes de lesiones de la piel. Durante el último año, los estudiantes tienen tres evaluaciones teóricas (una cada semana), de contenido acumulado, sobre las materias impartidas en el año anterior.

Para que el estudiante desarrolle la adecuada selección de pacientes en los servicios de atención primaria y conduzca adecuadamente los tratamientos que se pueden realizar en estas unidades, las actividades incluyen la observación de las lesiones de la piel y las prácticas de cuidado. Se desarrollan en el módulo multidisciplinario de Asistencia Integral a la Salud de Adultos, en 
el quinto año del curso, y lo constituyen 25 períodos, distribuidos en 15 días de trabajo de este módulo. Así, grupos de siete estudiantes, que ya habían recibido formación teórica y práctica en el año anterior, vinieron a ayudar a los pacientes en el Centro de Salud Jardim São José, una de las 47 UBS de la ciudad. Esta UBS es responsable de atender a cerca de 60.000 habitantes con bajos ingresos, en su mayoría formada por inmigrantes de otras regiones del país.

En este período, los estudiantes pasan por las etapas de Dermatología y Enfermedades de Transmisión Sexual (ETS). En el capítulo de ETS, el objetivo es permitir a los estudiantes el diagnóstico y tratamiento de estas enfermedades a través de la enseñanza integrada de las diversas especialidades relacionadas, incluyendo la Dermatología. En el capítulo Dermatología se espera que los estudiantes conozcan y traten las dermatosis más frecuentes en la región; también se enseñan conocimientos generales de la cirugía dermatológica, permitiendo realizar los procedimientos necesarios para el desempeño del médico de formación generalista.

Cada mañana, en la UBS, se atiende la demanda espontánea de los casos de ETS, y en tres períodos semanales también se atienden los casos de dermatología general. En uno de estos periodos se concentran los casos de enfermedad de Hansen. Dos mañanas a la semana se produce la participación de docentes de las áreas de Ginecología e Infectología en la atención de las ETS. En tres tardes, los estudiantes tienen las actividades clínicas y quirúrgicas en el Departamento de Dermatología, Hospital de Clínicas FCM-Unicamp; en otros dos períodos de práctica realizan procedimientos de cirugía menor en una UBS de Sumaré, una ciudad de la región, y en otro período, asisten al ambulatorio de Infecciones Ginecológicas en el Centro de Atención Integral a la Salud de la Mujer (CAISM/Unicamp).

Tras un año de aplicación de la reforma curricular, entre las 80 evaluaciones anónimas y voluntarias de estudiantes de quinto grado en el nuevo plan de estudios, 68 (85\%) calificaron la formación práctica como de importante para su formación, y 12 (15\%), como de importancia regular. Sesenta y tres de ellos $(78,75 \%)$ consideraban la práctica buena/excelente, $14(17,5 \%)$ regular y tres $(3,75 \%)$ pobre.

El objetivo de este estudio fue evaluar la capacidad médica en el tratamiento adecuado de pacientes con lesiones dermatológicas más prevalentes, las que tienen potencial de malignidad o las que pueden asociarse a una enfermedad sistémica. Se evaluaron las capacidades de médicos no especialistas del Programa de Salud de la Familia y de estudiantes de medicina de la FCM/ Unicamp, que habían frecuentado dos planos de estudio diferentes.

\section{Sujetos y métodos}

En este estudio, aprobado por el Comité de Ética de Investigación de la FCM/Unicamp, hemos elaborado una prueba escrita con preguntas de opción múltiple, que consta de diez casos clínicos y sus respectivas imágenes. Todas las preguntas fueron desarrolladas por uno de los profesores de Dermatología, que enseñó el mismo contenido académico a los estudiantes de ambos curriculares (antes y después de la reforma). Posteriormente, las preguntas se discutieron con otros dos autores y se efectuaron cambios para hacer más claro el texto. Todas las pruebas versaban sobre dermatosis prevalentes, cuyo conocimiento debe formar parte de la educación general de cualquier médico. El enfoque favorecía cuestiones prácticas sobre el razonamiento diagnóstico y el tratamiento de los casos, y no sólo sobre contenidos teóricos.

La prueba escrita se aplicó en cinco muestras diferentes, seleccionadas al azar. Uno de los autores supervisó la aplicación presencial de la prueba escrita en los siguientes grupos:

- Médicos no dermatólogos de cinco UBS del municipio de Campinas. Estas UBS habían sido seleccionadas por los coordinadores de salud de la ciudad para otro estudio [3] $(n=24)$.

- Médicos no especialistas que trabajaban en UBS en diferentes ciudades de los Departamentos Regionales de Salud (DRS) 7 y 14 del Estado de Sao Paulo y que asistieran a cursos de actualización en Clínica Médica en la FCM/ Unicamp $(n=51)$.

- Estudiantes de quinto año de licenciatura de medicina de la FCM/Unicamp que integraran el plan de estudios del nuevo modelo de curso, después de la reforma educativa $(n=24)$.

- Estudiantes de sexto año de medicina de la FCM/Unicamp que integraran el modelo antiguo del plan de estudios $(n=31)$. 
Tabla. Comparación entre errores y aciertos en cada grupo.

\begin{tabular}{lccccc}
\hline & Errores & Aciertos & OR & IC 95\% & $p$ \\
\hline Médicos & 595 & 155 & 2,52 & $1,82-3,48$ & \\
\hline 6. ${ }^{\circ}$ año & 236 & 74 & 2,09 & $1,42-3,07$ & $<0,001$ \\
\hline 5. ${ }^{\circ}$ año & 145 & 95 & 1,00 & & \\
\hline
\end{tabular}

OR: odds ratio; IC 95\%: intervalo de confianza al 95\%.

- Dermatólogos de la Sociedad Brasileña de Dermatología Regional del Estado de Sao Paulo, Distrito $5(n=8)$.

El enfoque de las preguntas se centró en enfermedades prevalentes: reacción a fármacos, eccemas dishidróticos, onicodistrofia, escabiosis, enfermedad de Hansen, sífilis... Además, se dio enfoque al conocimiento de las neoplasias y sus diagnósticos diferenciales: carcinoma de células basales e hiperplasia sebácea, carcinoma de células escamosas, y queratoacantoma y lesiones pigmentadas. En un caso de fotodermatitis se esperó a considerar la posibilidad diagnóstica de lupus eritematoso.

La cuestión relativa a la interpretación de la serología de sífilis se presentó y analizó en otro estudio [6].

Las pruebas fueron revisadas, sin identificación, por un docente de la disciplina de Dermatología de la FCM/Unicamp. Cada cuestión de la prueba escrita valía un punto; por lo tanto, el valor máximo que se obtenía era de sería diez. La nota media obtenida por el grupo de dermatólogos se consideró la puntuación máxima que se podría esperar, validando la herramienta de evaluación utilizada. Las notas de los demás grupos se analizaron estadísticamente en relación con ella. Se compararon las puntuaciones obtenidas por las cinco muestras.

\section{Resultados}

La prueba escrita fue contestada por 24 médicos de Campinas, 29 médicos de otras ciudades del
DRS 7, 22 médicos del DRS 14, 24 alumnos de quinto grado, 31 estudiantes de sexto año y ocho dermatólogos de la Sociedad Brasileña de Dermatología, que sirvieron como referencia.

Los alumnos de quinto grado obtuvieron una puntuación media de 6,34 en relación con la puntuación de los dermatólogos; los alumnos de sexto año, 3,82, y los médicos no especialistas de la red. 3,32. Los médicos de Campinas obtuvieron 3,80; los de otras ciudades del DRS 7, 3,15, y los del DRS 14, 3,00.

Los datos presentados en la tabla muestran que hubo significación estadística en la mejora de la adquisición de conocimientos en Dermatología en el nuevo plan de estudios de la FCM/Unicamp $(p<0,001)$; la posibilidad de un estudiante del currículo antiguo de errar en relación con los estudiantes del nuevo plan de estudios era 2,09 veces superior, y la de los médicos que trabajaban en Programas de Salud de la Familia, 2,52 veces superior.

\section{Discusión}

La consulta de pacientes con problemas dermatológicos es alta en las UBS [4,17-20]. Un gran número de médicos no dermatólogos se sienten inadecuadamente entrenados en la atención de pacientes con molestias o lesiones de la piel [1012,20]. Los gastos generados por terapéuticas erróneas, solicitud excesiva de pruebas diagnósticas y remisión a especialistas deben tomarse en consideración en la organización del sistema de salud [21]. 
Sin embargo, es responsabilidad de las instituciones de enseñanza médica una cualificación mínima necesaria para el desempeño del profesional de formación general y para los especialistas que trabajan en UBS. Esta formación debe permitir una evaluación inicial adecuada que permita diagnosticar y tratar correctamente las dermatosis más prevalentes, identificar pacientes con manifestaciones cutáneas que sugieran posibles enfermedades sistémicas, y detectar aquellos con lesiones de riesgo y que deban remitirse a un dermatólogo para reducir la morbilidad y la mortalidad.

Aunque las dermatosis son frecuentes en la práctica de los médicos generalistas, pediatras, ginecólogos y médicos de familia que trabajan en UBS, la remisión al especialista denota una formación inadecuada en Dermatología de estos profesionales.

Los casos clínicos presentados se relacionaban con enfermedades prevalentes, dermatosis susceptibles de transformación maligna o dermatosis con un diagnóstico diferencial de neoplasias. Todos estos asuntos son importantes en la práctica médica.

Entre los grupos participantes en el estudio, los estudiantes que siguieron el nuevo plan de estudios de la FCM/Unicamp fueron aquellos cuyo desempeño más se parecía al mostrado por los dermatólogos, lo que expresava una mejor formación en la especialidad.

Los estudiantes de sexto año y los médicos de la UBS Campinas obtuvieron resultados comparables $(3,82$ y 3,80 , respectivamente). El promedio de los 75 médicos no especialistas formados por diferentes facultades de medicina del país fue de 3,32.

Aunque sea escasa la bibliografía sobre el impacto de reformas curriculares de los estudios de medicina en la enseñanza de la Dermatología, los datos aquí mostrados son consistentes con los hallazgos de otros estudios con diseños similares y refuerzan la importancia de una reevaluación constante de la educación [2,22-26].

Una limitación del estudio fue el escaso número de participantes. Las perspectivas de futuro incluyen la aplicación de la prueba escrita en grupos mayores de médicos (especialistas y no especialistas) y nuevas búsquedas para evaluar, entre los graduados de ambos modelos de planes de estudio, la relevancia de la Dermatología y la seguridad en el diagnóstico y tratamiento de los pacientes con dermatosis atendidos por ellos.

En conclusión, el estudio permitió demostrar que los cambios en la enseñanza de la Dermatolo- gía fueron importantes para la adquisición de conocimientos de la especialidad por los estudiantes que asistieron al nuevo plan de estudios. También permitió suponer que los médicos no especialistas del Programa de Salud Familiar que prestan servicios en las UBS estudiadas no recibían formación suficiente para el abordaje de la especialidad como médicos de formación general.

\section{Bibliografía}

1. Carli P, Giorgi V, Palli D, Maurichi A, Mulas P, Orlandi C, et al. Dermatologist detection and skin self-examination are associated with thinner melanomas -results from a survey of the Italian Multidisciplinary Group on Melanoma. Arch Dermatol 2003; 139: 607-12.

2. Burge S; British Association of University Teachers of Dermatology. Teaching dermatology to medical students: a survey of current practice in the U.K. Br J Dermatol 2002; 146: 295-303.

3. Yutronic J, Leiva A, Espinoza M, Bello MP, Correa F, Saavedra T, et al. Adult skin disorders encountered at the emergency department of the Clinical Hospital University of Chile. In: International League of Dermatological Societies. Proceedings of the 21st World Congress of Dermatology; 2007 Sep 30-Oct 5; Buenos Aires, Argentina. eQip Abstracts on CD; 2007. 5960.

4. Greco P, Cafiero P, Rípoli M, Pierini AM. Proporción de patología dermatológica en la atención ambulatoria pediátrica. Arch Argent Dermatol 1997; 47: 91-7.

5. Santos A Jr, Andrade MGG, Zeferino AB, Monte Alegre S, Moraes AM, Velho PENF. Prevalência de dermatoses na rede básica de Campinas, São Paulo, Brasil. An Bras Dermatol 2007; 82: 419-24.

6. Halpern A. Are primary care physicians well trained in dermatology? Manag Care 1995; 4: 49.

7. Clayton R, Perera R, Burge S. Defining the dermatological content of the undergraduate medical curriculum: a modified Delphi study. Br J Dermatol 2006; 155: 137-44.

8. Kirsner RS, Federman DG. Lack of correlation between internists' ability in dermatology and their patterns of treating patients with skin disease. Arch Dermatol 1996; 132: 1043-6.

9. Santos A Jr, Andrade MGG, Magalhães RF, Moraes AM, Velho PENF. Sorologia para sífilis: os médicos estão capacitados a interpretá-la? An Bras Dermatol 2007; 82: 183-5.

10. Barbarulo AM, Gavazza S, Fontana MI, Berbari S, Azcune 
R. Evaluación de la capacidad diagnóstica de los médicos generales en dermatología. Arch Argent Dermatol 2002; 52: 111-5.

11. Whitaker-Worth DL, Susser WC, Grant-Kels JM. Clinical dermatologic education and the diagnostic acumen of medical students and primary care residents. Int J Dermatol 1998; 37: 855-9.

12. Stephenson A, From L, Cohen A, Tipping J. Family physicians' knowledge of malignant melanoma. J Am Acad Dermatol 1997; 37: 953-7.

13. Hymowitz MB, Hayes BB, Maury JJ, Geller AC. Evaluation of medical students' knowledge, attitudes, and personal practices of some protection and skin self-examination. Arch Dermatol 2006; 142: 523-4.

14. Moore MM, Geller AC, Zhang Z, Hayes BB, Bergstrom K, Graves JE, et al. Skin cancer examination teaching in US medical education. Arch Dermatol 2006; 142: 439-44.

15. Mahé A, Faye O, N’Diaye HT, Konaré HD, Coulibaly I, Kéita S, et al. Integration of basic dermatological care into primary health care services in Mali. Bull World Health Organ 2005; 83: 935-41.

16. BAUTOD. Teaching recommendations for undergraduate dermatology in the UK. London: British Association of Dermatologists; 2000.

17. Royal College of General Practitioners. Morbidity statistics from general practice: fourth national study 1991-92. London: HSMO; 1995.
18. Julian CG. Dermatology in general practice. Br J Dermatol 1999; 141: 518-20.

19. Lowell BA, Froelich CW, Federman DG, Kirsner RS. Dermatology in primary care: prevalence and patient disposition. J Am Acad Dermatol 2001; 45: 250-5.

20. Solomon BA, Collins R, Silverberg NB, Glass AT. Quality of care: issue or oversight in health care reform? J Am Acad Dermatol 1996; 34: 601-7.

21. Federman D, Hogan D, Taylor JR, Caralis P, Kirsner RS. A comparison of diagnosis, evaluation, and treatment of patients with dermatologic disorders. J Am Acad Dermatol 1995; 32: 726-9.

22. Burge SM, Lancaster T. Assessment in undergraduate dermatology. Clin Exp Dermatol 2004; 29: 441-6.

23. Doshi DN, Firth K, Mintz M, Ehrlich A. Pilot study of a skin cancer education curriculum for medical students. J Am Acad Dermtol 2007; 56: 167-9.

24. Enk CD, Gilead L, Smolovich I, Cohen R. Diagnostic performance and retention of acquired skills after dermatology elective. Int J Dermatol 2003; 42: 812-5.

25. Al-Hoqail IA, Gad A, Crawford RI. Dermatology practice in primary health care services: where do we stand in the Middle East? Int J Dermatol 2002; 41: 4-7.

26. Finlay AY, Coles EC, Dawber RP, Graham-Brown RA, Hunter JA, Marks JM. Dermatology examination performance: wide variation between different teaching centres. Med Educ 1994; 28: 301-6. 